\title{
Aktuelle Studien bei Schizophrenie und Demenz: Körperpsychotherapie, Tanz-, Bewegungstherapie und Psychomotorik
}

\author{
Iris Bräuninger
}

\section{Reduktion der Negativsymptomatik bei Schizophrenie durch Körperpsycho- therapie und Tanz-, Bewegungstherapie: eine randomisierte kontrollierte Studie}

Schizophrenie kann Ausdruck eines Disembodiments oder eines gestörten Embodiment des Selbst sein. Disembodiment ist nach Martin et al. (2016) „the disturbance and alienation of the habitual or implicit bodily functioning on the level of perception, action or intercorporeality.“ (S.2) Auf dieser Theorie basiert die Annahme, dass sich negative Symptome bei Schizophrenie mit auf Embodiment fokussierten Ansätzen wie Körperpsychotherapie (BPT) und Tanz-, Bewegungstherapie (DMT) reduzieren lassen (Martin et al. 2016). Die randomisierte kontrollierte Studie mit Doppelverblindung der Rater untersuchte die Wirksamkeit der manualisierten Bewegungstherapie (BPT / DMT) auf negative Symptome von PatientInnen mit Schizophrenie. Doppelverblindung bedeutet, dass die Rater nicht wussten, welche Teilnehmerlnnen welcher Gruppe zugeteilt wurden. Die erste Hypothese ging davon aus, dass sich negative Symptome generell durch BPT / DMT reduzieren ließen, wenn gleichzeitig extrapyramidale Nebeneffekte der antipsychotischen Medikation kontrolliert würden. Das globale Level negativer Symptome wurde mit der Skala SANS (Scale for the Assessment of Negative Symptoms) erfasst. Die zweite Hypothese erwartete einen spezifischen positiven Effekt der BPT / DMT auf nicht-kognitive negative Symptome wie den Verlust emotionaler Schwingungsfähigkeit (blunted affect). Blunted Affect bringen die Autorlnnen in Zusammenhang mit Disembodiment des Selbst.

Die 68 ambulanten Schizophrenie-PatientInnen (Durchschnittsalter 39.84 Jahre, SD = 10.35, Länge der durchschnittlichen Erkrankung 15.92 Jahre, $\mathrm{SD}=10.00$ ) wurden auf zwei Behandlungsgruppen DMT / BP $(n=44)$ und auf eine Warte-Kontrollgruppe $(n=24)$ randomisiert. Alle drei Gruppen erhielten die übliche Behandlung (,Treatment as Usual“ - TAU). Die beiden Behandlungsgruppen nahmen zusätzlich über zehn Wochen an einer ambulanten 20-stündigen manualisierten Bewegungstherapie-Gruppenintervention teil. Von den drei- und sechs-monatigen Follow-up-Untersuchungen wurde aufgrund der Dropout-Rate von 31\% abgesehen. Am Ende der BPT / DMT-Intervention wies die Interventionsgruppe signifikant weniger negative Symptome im Vergleich zur Warte-Kontrollgruppe auf, und zwar bei der SANS-Gesamtbewertung (SANS-TS: $\mathrm{t}(66)=-2.70, \mathrm{p}<0.01)$ und den Unterwerten Blunted Affect (SANS-BA: $\mathrm{t}(66)=-2.22$, $p<0.05$ ), Freudlosigkeit (SANS-Anhedonia: t(66) $=-2.37, p<0.05$ ) und Aufmerksamkeit (SANS-Attention: $\mathrm{t}(66)=-2.70, \mathrm{p}<0.01)$. Die Autorlnnen schlussfolgern, dass Körperpsychotherapie und Tanztherapie sehr wirksam seien, um die Negativsymptomatik bei PatientInnen mit Schizophrenie zu reduzieren, und schlagen deshalb vor, dass dieser Ansatz in der Routinebehandlung fest verankert werden sollte.

\section{Wirkung einer Psychomotorik/Tanz-} therapie-Intervention auf Stimmung und Verhalten von DemenzpatientInnen

Die zweite Studie von Guzmán und KollegInnen (2016) überprüfte die Wirkung der manualisierten Psychomotorik/Tanztherapie-Intervention DANCIN auf die Stimmung und das Verhalten von Pflegeheimbewohnern mit leichter bis mittlerer Demenz. Als Intervention wurde Latin Ballroom (Danzón) durchgeführt, und als Methode wurde 
eine Multiple-Baseline-Einzelfall-Studie in drei Phasen A-B-C angewandt: Phase A enthielt gestaffelte Baselines unterschiedlicher Längen (drei bis sechs Wochen). Phase B bestand aus einer 12-wöchigen DANCIN-Intervention. Für Phase C wurde eine Nachuntersuchung 12 Wochen nach Beendigung der Intervention (nach Phase B) durchgeführt. Das Multiple-Baseline-Einzelfall-Design ermöglichte, dass die Teilnehmerlnnen als ihre eigene Kontrollgruppe für die Effekte von DANCIN zwischen Phase A und B fungierten. An der Studie nahmen zehn TeilnehmerInnen (sechs Frauen und vier Männer) aus zwei Pflegewohnheimen und einem Wohnheim teil. Leitende Mitarbeiterlnnen $(n=32)$ überwachten, dass die Teilnehmerlnnen Tagebücher führten, und sie erfüllten unterschiedliche Aufgaben bei der Evaluation und der Protokollierung der quantitativen Daten. Als Outcome-Messinstrument wurde die Dementia Mood Assessment Scale DMAS-17 eingesetzt. Zur Datenanalyse wurde ein nicht-parametrisches statistisches Verfahren angewandt.

Die Ergebnisse zeigten eine kleine bis mittlere Verbesserung in 21 von 32 Stimmung/VerhaltenItems, acht Items zeigten keine Veränderung, und drei Items zeigten in einem Fall negative Wirkungen. Die Autorlnnen wiesen auf methodische Schwächen ihrer Studie hin. Sie schlussfolgerten, dass die Studie die wachsenden Beweise für körperorientierte psychologische Therapien bei leichten und mittelschweren Stadien der Demenz unterstützt. Psychomotorik/Tanztherapie habe das Potential, positive Verhaltensänderung und Verbesserung der Stimmung hervorzurufen, wie beispielsweise die Reizbarkeit zu verringern und das Selbstwertgefühl zu verbessern.

\section{Schlussfolgerung}

In der ersten Studie (Martin et al. 2016) zeigt sich die Behandlungsgruppe Körperpsychotherapie und Tanztherapie für die Reduktion der Negativsymptomatik bei Schizophrenie gegenüber der Kontrollgruppe als klinisch relevant. Die Studie erfüllte die Anforderung für eine „Treatment as Usual
(TAU) “-Kontrollgruppe, wie sie u. a. bei Priebe und KollegInnen (2016) formuliert wurde. Ferner folgt sie dem Gold-Standard nach RCTs. Sie bestätigt die Ergebnisse eines RCT zur Wirksamkeit körperorientierter Psychotherapie auf die negativen Symptome bei Schizophrenie (Röhricht/Priebe 2006). Martin und KollegInnen versuchten, Verzerrungen durch die hohe Dropout-Rate von 31\% mithilfe von Multiple Imputation (MI) zu kontrollieren. Zukünftige Studien könnten die Personen untersuchen, die frühzeitig aufhören würden. Eine Vermutung wäre, dass es sich bei dieser Untergruppe um Personen mit besonders stark ausgeprägter Negativsymptomatik handeln könnte. Interessant wäre zu untersuchen, ob spezifische tanztherapeutische Interventionen die Behandlungscompliance verbessern und die Abbruchrate senken könnten. Die vorgestellte Studie bietet einen wichtigen Beitrag zur Evidenzbasierung der Körperpsychotherapie und Tanztherapie bei Schizophrenie. Darüber hinaus präsentiert sie mit der manualisierten BPT/DMT spezifische Interventionen, die sich als erfolgreich zur Reduktion von Negativsymptomatik erwiesen haben.

Die zweite Studie von Guzmán und KollegInnen (2016) zur Psychomotorik-Tanztherapie-Intervention DANCIN weist methodische Schwachstellen auf. Dies verwundert insofern nicht, da randomisierte kontrollierte Studien in diesem Bereich grundsätzlich sehr aufwendig sind, lange Studienlaufzeiten beinhalten mit Schwierigkeiten beim Erhalt des informed consent und mit hohen Dropout-Raten gerechnet werden muss. Die Klassifikation der Intervention als „Tanztherapie“ wirft Fragen nach der Definition auf. Die Absicht der Autorlnnen, mit ihrer Studie die Basis für eine größere kontrollierte Machbarkeitsstudie zu legen, erscheint sinnvoll. Allerdings sollten zukünftige Studien nicht eng auf diesen sehr spezifischen Ansatz DANCIN beschränkt bleiben. Vielmehr könnten sie, dem Beispiel der Studie von Martin und Kolleglnnen folgend, einen umfassenderen Ansatz wie BPT / DMT berücksichtigen.

In beiden hier vorgestellten klinischen Bereichen wie der Behandlung von Schizophrenie und 
Demenz mit Tanz-, Bewegungstherapie und Körperpsychotherapie wären weitere Studien mit RCT-Design, großer Teilnehmerlnnenzahl und methodisch hoher Studienqualität für eine starke statistische Aussagekraft anzustreben. Zukünftig vermehrt multizentrische deutschlandweite oder europäische Studien zu planen und durchzuführen, wäre eine Möglichkeit. Dies würde zwar einen hohen organisatorischen und koordinatorischen Aufwand bedeuten. Bundesweit und europäisch vernetzt mit höheren Studien-TeilnehmerInnenzahlen und größerer statistischer Aussagekraft von RCTs zu denken, zu planen und zu forschen, würde die wissenschaftliche Fundierung dieser Ansätze weiter vorantreiben.

\section{Literatur}

Guzmán, A., Freeston, M., Rochester, L., Hughes, J. C., James, I. A. (2016): Psychomotor Dance Therapy Intervention (DANCIN) for people with dementia in care homes: a multiple-baseline single-case study. International psychogeriatrics (First View), 1-21, http://dx.doi.org/10.1017/s104161021600051x

Martin, L. A., Koch, S. C., Hirjak, D., Fuchs, T. (2016): Overcoming Disembodiment: The Effect of Movement Therapy on Negative Symptoms in Schizophrenia - A Multicenter Randomized Controlled Trial. Frontiers in psychology 7 (483), http://dx.doi. org/10.3389/fpsyg.2016.00483

Priebe, S., Savill, M., Wykes, T., Bentall, R., Lauber, C., Reininghaus, U., McCrone, P., Mosweu, P., Bremner, S., Eldridge, S., Röhricht, F. (2016): Clinical effectiveness and cost-effectiveness of body psychotherapy in the treatment of negative symptoms of schizophrenia: a multicentre randomised controlled trial. Health Technology Assessment 20 (11), 1-100, http://dx.doi.org/10.3310/hta20110

Röhricht, F., Priebe, S.(2006): Effect of body oriented psychological therapy on negative symptoms in schizophrenia: a randomized controlled trial. Psychological Medicine 36, 669-678, http://dx.doi. org/10.1017/So033291706007161

\section{Die Autorin}

\section{Dr. Iris Bräuninger}

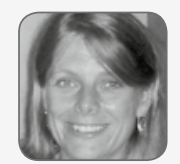

Wissenschaftliche Mitarbeiterin

Psychiatrische Universitätsklinik

Zürich, Dozentin im Masterstudiengang Tanztherapie an der Autonomen Universität Barcelona

(UAB), Supervisorin, Ausbilderin und Lehrtherapeutin der deutschen und spanischen Berufsverbände (BTD, ADMTE), Kestenberg Bewegungsnotatorin, Psychotherapie (ECP), Private Praxis für Supervision und Therapie Bodensee.
Dr. Iris Bräuninger

Psychiatrische Universitätsklinik Zürich

Direktion Pflege, Therapien und Soziale Arbeit

Forschung und Entwicklung

Lenggstr. $31 \mid \mathrm{CH}-8032$ Zürich

Tel. +41 (0) 44-384 2717

+41(0)77-4422676

iris.braeuninger@puk.zh.ch oder

dancetherapy@mac.com 